\title{
Distribution of betulinic acid in plant kingdom
}

\author{
S. R. Pai ${ }^{* \otimes}$ and R. K. Joshi ${ }^{*} \otimes$
}

\begin{abstract}
Betulinic acid (3ß-hydroxy-lup-20(29)-en-28-oic acid) is a ubiquitous pentacyclic triterpenoid found in the plants. It is highly valued for its role in wide array of ailments viz. anti-HIV, anti-malarial, anti-cancerous, hepatoprotective, and many more. In lights of tremendous interest in recent years on chemistry and pharmacological properties of betulinic acid (BA), comprehensive data have been collected in this review to present its distribution in plant kingdom.
\end{abstract}

Keywords: Betulinic acid; Triterpenoids

\section{Introduction}

Plants are well known for their medicinal value with the finding of cinchona in $17^{\text {th }}$ century, followed by digitoxin, morphine, introduction of synthetic asprin, a derivative of a plant-based drug, are wonders of the diverse floristic wealth (Raskin \& Ripoll, 2004). Natural products offer large structural diversity and techniques for separation, structure elucidation, screening and combinatorial synthesis have led to revitalization of these secondary metabolites as sources of new drugs (Saklani \& Kutty, 2008).

Received: 13 May 2014 / Accepted: 19 June 2014 / Published online: 2 July 2014

(C) Horizon e-Publishing Group

CITATION

Pai, S. R., \& Joshi, R. K. (2014). Distribution of betulinic acid in plant kingdom. Plant Science Today, 1(3), 103-107. http://dx.doi.org/10.14719/pst.2014.1.3.58

\section{AUTHORS' AFFILIATION}

${ }^{1}$ Department of Plant Biotechnology and Tissue Culture, Regional Medical Research Centre (RMRC), Indian Council of Medical Research (ICMR), Nehru Nagar, Belgaum - 590 010, Karnataka State, India.

2 Department of Phytochemsitry, Regional Medical Research Centre (RMRC), Indian Council of Medical Research (ICMR), Nehru Nagar, Belgaum - 590 010, Karnataka State, India.

* Both the authors have contributed equally.

■CORRESPONDENCE: S. R. Pai, Email: drpaisr@gmail.com; R. K. Joshi, Email: joshirk_natprod@yahoo.com
Betulinic acid (BA) (3ß-hydroxy-lup-20(29)-en-28-oic acid molecular formula: $\mathrm{C}_{30} \mathrm{H}_{48} \mathrm{O}_{3}$ and molecular weight: 456.7) is a pentacyclic triterpenoid (Fig. 1). It is moderately soluble in water and relatively nontoxic, found in many plants, especially in tree species. It is a biologically active compound, mainly known for its selective inhibitor of human melanoma (Pisha et al., 1995). The compound gains its name because of its prevalence in the family Betulaceae, which includes Betula alba, B. pubescens, B. platyphylla B. maximowicziana, B. mandshurica and others. The family still serves as a major source of betulinic acid. Also its congener betulin, is one of the first natural products isolated in 1788 from the bark of white birch, Betula alba (Krasutsky, 2006).

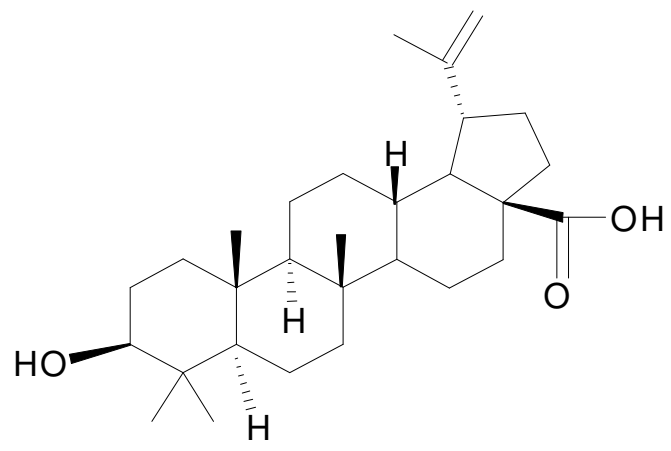

Fig 1. Chemical Structure of betulinic acid

Search tools including, Google Scholar $\AA$, PubMed $\AA$ and Scopus $\AA$ were used to generate the literature on BA. Over $\sim 800$ hits were spotted which are related to betulinic acid. Out of which the reports related to the subject matter of this review were considered.

\section{Distribution of BA in plant species}

Betulinic acid is widely distributed in nature and its occurrence across a wide multitude of taxonomically different genera has been reported. Therefore, in this review, attempt has been made to list the plant species in which the compound BA has been accounted (Table 1). 
Table 1. Distribution of betulinic acid in various plant species

\begin{tabular}{|c|c|c|c|}
\hline Plant name & Family & Part & Reference \\
\hline Achyranthes aspera & Amaranthaceae & Leaf & Pai, Upadhya, Hegde, Joshi, \& Kholkute, 2014 \\
\hline Ampelozizyphus amazonicus & Rhamnaceae & Stem & Rosas et al., 2007 \\
\hline Ancistrocladus heyneanus & Ancistrocladaceae & Roots & Bringmann et al., 1997 \\
\hline Ancistrocladus heyneanus & Ancistrocladaceae & Leaf & Pai, Nimbalkar, Pawar, \& Dixit, 2011 \\
\hline Arbutus menziesii & Ericaceae & Stem Bark & Yogeeswari \& Sriram, 2005 \\
\hline Berlinoa grandiflora & Leguminoceae & Stem Bark & Yogeeswari \& Sriram, 2005 \\
\hline Betula platyphylla & Betulaceae & Stem Bark & Zhang, Yu, \& Wang, 2003 \\
\hline Betula pubescens & Betulaceae & Stem Bark & $\begin{array}{l}\text { Abyshev, Zhurkovich, Agaev, Abdulla-zade, \& Guseinov, } \\
\text { 2006; } \\
\text { Abyshev, Agaev, \& Guseinov, } 2007\end{array}$ \\
\hline Betula pendula & Betulaceae & Stem Bark & $\begin{array}{l}\text { Holonec, Ranga, Crainic, Truta, \& Socaciu, 2012; } \\
\text { Galgon, Hoke, \& Drager, } 1999\end{array}$ \\
\hline Betula maximowicziana & Betulaceae & Stem Bark & Abyshev et al., 2007 \\
\hline Betula mandshurica & Betulaceae & Stem Bark & Abyshev et al., 2007 \\
\hline Caesalpinia paraguariensis & Fabaceae & Arial & Woldemichael, Singh, Maiese, \& Timmermann, 2003 \\
\hline Callicarpa macrophylla & Lamiaceae & Herb & Pan, Jia, \& Sun, 2008 \\
\hline Cichorium intybus & Asteraceae & Seeds & Atta-ur-Rahman, Zareen, Choudhary, Akhtar, \& Khan, 2008 \\
\hline Clerodendrum mandarinorum & Verbenaceae & Root & Zhu, Phillipson, Greengrass, \& Bowery, 1996 \\
\hline Coussarea paniculata & Rubiaceae & Stem & Chaturvedula, Schilling, Johnson, \& Kingston, 2003 \\
\hline Diospyros leucomelas & Ebenaceae & Stem & Recio et al., 1995 \\
\hline Diospyros melanoxylon & Ebenaceae & Stem & Kantamreddi \& Wright, 2007 \\
\hline Diospyros peregrina & Ebenaceae & Stem & Kantamreddi \& Wright, 2007 \\
\hline Diospyros sylvatica & Ebenaceae & Stem & Kantamreddi \& Wright, 2007 \\
\hline Diospyros tomentosa & Ebenaceae & Stem & Kantamreddi \& Wright, 2007 \\
\hline Dichapetalum gelonioides & Dichapetalaceae & Stem Bark & Fang et al., 2006 \\
\hline Dillenia papuana & Dilleniaceae & $\begin{array}{l}\text { Leaves, } \\
\text { Stem }\end{array}$ & Nick, Wright, Rali, \& Sticher, 1995 \\
\hline Doliocarpus schottianus & Dilleniaceae & Arial & De Oliveira, Santos, \& Espindola, 2002 \\
\hline Eucalyptus camaldulensis & Myrtaceae & Leaves & Begum, Sultana, Siddiqui, Shaheen, \& Gilani, 2002 \\
\hline Henriettella fascicularis & Melastomataceae & Stem & Calderon et al., 2002 \\
\hline Ipomea pescaprae & Convolvuleaceae & Root bark & Yogeeswari \& Sriram, 2005 \\
\hline Licania arianeae & Chrysobalanaceae & $\begin{array}{l}\text { Stem, } \\
\text { Leaves }\end{array}$ & De Carvalho et al., 2008 \\
\hline Melaleuca leucadendron & Myrtaceae & Leaves & Lee, 1998 \\
\hline Morus alba & Moraceae & Stem, Root & Nattapong and Omboon, 2008 \\
\hline Morus australis & Moraceae & Roots & Ko, Yu, Ko, Teng, \& Lin, 1997 \\
\hline Nepeta nuda & Lamiaceae & Arial & Kokdil, Yalçin, \& Topçu, 1999 \\
\hline Nerium oleander & Apocynaceae & Leaves & Fu et al., 2005 \\
\hline Oenothera biennis & Onagraceae & Arial & Hamburger et al., 2002 \\
\hline Paeonia suffruticosa & Ranunculaceae & Roots & Lin, Ding, \& Wu, 1998 \\
\hline Physocarpus intermedium & Rosaceae & Stem Bark & Yogeeswari \& Sriram, 2005 \\
\hline Prunus dulcis & Rosaceae & Hull & Takeoka et al., 2000 \\
\hline Quercus suber & Fagaceae & Stem Bark & Sousa, Pinto, Silvestre, \& Neto, 2006 \\
\hline Rosmarinus officinalis & Lamiaceae & $\begin{array}{l}\text { Arial } \\
\text { Leaves }\end{array}$ & R azborek, Voncina, Dolecek, \& Voncina, 2007; 2008 \\
\hline Salvia officinalis & Lamiaceae & Leaves & Razborek et al., 2008 \\
\hline Salvia glutinosa & Lamiaceae & Leaves & Razborek et al., 2008 \\
\hline Salvia sclarea & Lamiaceae & Leaves & Razborek et al., 2008 \\
\hline Satureja montana & Lamiaceae & Leaves & Razborek et al., 2008 \\
\hline Strychnos vanprukii & Loganiaceae & Arial & Chien et al., 2004 \\
\hline Syncarpa glomulifera & Myrtaceae & Stem Bark & Yogeeswari \& Sriram, 2005 \\
\hline Syzigium claviflorum & Myrtaceae & Leaves & Fujioka et al., 1994 \\
\hline Syzigium formasanum & Myrtaceae & Leaves & Chang, Wu, Hsieh, Kuo, \& Lee Chao, 1999 \\
\hline Tabernaemontana cathariensis & Apocynaceae & Root & Pereira et al., 2008 \\
\hline Tetracentron sinense & Trochodendraceae & Stem bark & How, Wu, Ko, \& Chen, 1982 \\
\hline Triphyophyllum peltatum & Dioncophyllaceae & Roots & Bringmann et al., 1997 \\
\hline Ugni molinae & Myrtaceae & Leaves & Goity et al., 2013 \\
\hline Vitex negundo & Verbenaceae & Leaves & Yogeeswari \& Sriram, 2005 \\
\hline Vitex negundo & Verbenaceae & Leaves & Taralkar \& Chattopadhyay, 2012 \\
\hline Viscum album & Viscaceae & Arial & Jäger, Winkler, Pfüller, \& Scheffler, 2007 \\
\hline Ziziphus jujuba & Ramnaceae & Fruits & Guo et al., 2009 \\
\hline Zizyphus xylopyrus & Ramnaceae & Stem & Jagadeesh, David Krupadanam, \& Srimannarayana, 1998 \\
\hline
\end{tabular}




\section{Acknowledgements}

Authors are indebted to Officer in-Charge, RMRC, Belgaum and Indian Council of Medical Research, New Delhi, India. SRP is grateful to SERB, Department of Science and Technology, New Delhi for providing financial assistance during the work (Grant No. SB/YS/LS-71/2013).

\section{References}

Abyshev, A. Z., Agaev, É. M., \& Guseinov, A. B. (2007). Studies of the chemical composition of birch bark Extracts (Cortex betula) from the Betulaceae family. Pharmaceutical Chemistry Journal, 41(8), 22-26. http://dx.doi.org/10.1007/s11094-007-0091-5

Abyshev, A. Z., Zhurkovich, I. K., Agaev, É. M., Abdulla-zade, A. A., \& Guseinov, A. B. (2006). Methods of standardization of the quality of betulenol parent substance and its ready-to-use medicinal forms. Pharmaceutical Chemistry Journal, 40(1). http://dx.doi.org/10.1007/s11094-006-0056-0

Atta-ur-Rahman, Zareen S., Choudhary, M. I., Akhtar, M. N., \& Khan, S. N. (2008). A-glucosidase inhibitory activity of triterpenoids from Cichorium intybus. Journal of Natural Products, $\quad 71, \quad 910-913,2008$ http://dx.doi.org/10.1021/np800001v PMid:18341288

Begum, S., Sultana, I., Siddiqui, B. S., Shaheen, F., \& Gilani, A. H. (2002). Structure and spasmolytic activity of eucalyptanoic acid from Eucalyptus camaldulensis var. obtusa and synthesis of its active derivative from oleanolic acid. Journal of Natural Products, 65, 1939-1941. http://dx.doi.org/10.1021/np020127x PMid:12502346

Bringmann, G., Saeb, W., Aké Assi, L., Francois, G., Sankara Narayanan, A. S., Peters, K., \& Peters, E. M. (1997). Betulinic acid: Isolation from Triphyophyllum peltatum and Ancistrocladus heyneanus, antimalarial activity, and crystal structure of the benzyl ester. Planta Medica, 63, 255 - 257. http://dx.doi.org/10.1055/s-2006-957666 PMid:9225608

Calderon, A. I., Terreaux, C., Schenk, K., Pattison, P., Burdette, J. E., Pezzuto, J. M., Gupta, M. P., \& Hostettmann, K. (2002). Isolation and structure elucidation of an isoflavone and a sesterterpenoic acid from Henriettella fascicularis. Journal of Natural Products, 65(12), 1749-1753. http://dx.doi.org/10.1021/np0201164 PMid:12502307

Chang, C., Wu, T., Hsieh, Y., Kuo, S., \& Lee Chao, P. (1999). Terpenoids of Syzygium formosanum. Journal of Natural Products, 62, 327-328 http://dx.doi.org/10.1021/np980313w PMid:10075776

Chaturvedula, V. S. P., Schilling, J. K., Johnson, R. K., \& Kingston, D. G. I. (2003). New cytotoxic lupane triterpenoids from the twigs of Coussarea paniculata. Journal of Natural Products, 66, 419-422. http://dx.doi.org/10.1021/np0204848 PMid:12662105

Chien, N. Q., Hung, N. V., Santarsiero, B. D., Mesecar, A. D., Cuong, N. M., Soejarto, D. D., Pezzuto, J. M., Fong, H. H. S., \& Tan, G. T. (2004). New 3-0-acyl betulinic acids from Strychnos vanprukii Craib. Journal of Natural Product, 67, 994-998. http://dx.doi.org/10.1021/np030469i PMid:15217281

De Carvalho, M. G., de Oliveira Cândido, L. F., da Costa, P. M., do Nascimento, I. A., \& Braz-Filho, R. (2008). Triterpenes acids and saponins isolated from Licania arianeae Prance (Chrysobalanaceae). Journal of Natural Medicine, 62, 122-123. http://dx.doi.org/10.1007/s11418-008-0241-4

De Oliveira, B. H., Santos, C. A. M., \& Espindola, A. P. D. M. (2002). Determination of the triterpenoid, betulinic acid, in Doliocarpus schottianus by HPLC. Phytochemical Analysis, 13: 95-98. http://dx.doi.org/10.1002/pca.628 PMid:12018029

Fang, L., Ito, A., Chai, H., Mi, Q., Jones, W. P., Madulid, D. R., Oliveros, M. B., Gao, Q., Orjala, J., Farnsworth, N. R., Soejarto, D. D., Cordell, G. A., Swanson, S. M., Pezzuto, J. M., \& Kinghorn, A. D. (2006). Cytotoxic constituents from the stem bark of Dichapetalum gelonioides collected in the Philippines. Journal of Natural Product, 69, 332-337. http://dx.doi.org/10.1021/np058083q PMid:16562829 PMCid:PMC2543135

Fu, L., Zhang, S., Li, N., Wang, J., Zhao, M., Sakai, J., Hasegawa, T., Mitsui, T., Kataoka, T., Oka, S., Kiuchi, M., Hirose, K., \& Ando, M. (2005). Three new triterpenes from Nerium oleander and biological activity of the isolated compounds. Journal of Natural Products, 68, 198-206.

Fujioka, T., Kashiwada, Y., Kilkuskie, R. E., Cosentino, L. M., Ballas, L. M., Jiang, J. B., Janzen, W. P., Chen, I. S., \& Lee, K. H. (1994). Anti-AIDS agents, 11, betulinic acid and platanic acid as anti-HIV principles from Syzigium claviflorum and the anti-HIV activity of structurally related triterpenoids. Journal of Natural Products, 57(2), 243-247. http://dx.doi.org/10.1021/np50104a008 PMid:8176401

Galgon, T., Hoke, D. \& Drager, B. (1999). Identification and quantification of betulinic acid. Phytochemical Analysis, 10(4), 187 - 190. http://dx.doi.org/10.1002/(SICI)1099-1565(199907/08)1 0:4<187::AID-PCA443>3.0.CO;2-K

Goity, L.E., Queupil, M. J., Jara, D., Alegría, S. E., Pe-a, M., Barriga, A., Aguirre, M. C., \& Delporte, C. (2013). An HPLC-UV and HPLC-ESI-MS based method for identification of anti-inflammatory triterpenoids from the extracts of Ugni molinae. Boletín Latinoamericano y del Caribe de Plantas Medicinales y Aromáticas, 12(1), 108 - 116.

Guo, S., Duan, J., Tang, Y., Su, S., Shang, E., Ni, S., \& Qian, D. (2009). High-performance liquid chromatography-two wavelength detection of triterpenoid acids from the fruits of Ziziphus jujuba containing various cultivars in different regions and classification using chemometric analysis. Journal of Pharmaceutical and Biomedical Analysis, 49(5), 1296-1302. http://dx.doi.org/10.1016/i.jpba.2009.03.006 PMid:19359121

Hamburger, M., Riese, U., Graf, H., Melzig, M. F., Ciesielski, S., Baumann, D., Dittmann, K., \& Wegner, C. (2002). Constituents in evening primrose oil with radical scavenging, cyclooxygenase, and neutrophil elastase inhibitory activities. Journal of Agriculture and Food Chemistry, 50, 5533-5538.

Holonec, L., Ranga, F., Crainic, D., Truta, A., \& Socaciu, C. (2012). Evaluation of betulin and betulinic acid content in birch bark from different forestry areas of Western Carpathians. Notulae Botanicae Horti Agrobotanici, 40(2), 99-105.

How, F. C., Wu, T. L., Ko, W. C., \& Chen, T. C. (1982). In: $A$ dictrionary of the families and genera of Chienese seed 
plants. Beijing: Science Press. pp 483, http://dx.doi.org/10.1039/b606816b PMid:17119640

Jagadeesh, S. G., David Krupadanam, G. L., \& Srimannarayana, G. (1998). Tobacco caterpillar antifeedent from the gotti stem wood triterpene betulinic acid. Journal of Agriculture and Food Chemistry, 46, 2797-2799. http://dx.doi.org/10.1021/if970768b

Jäger, S., Winkler, K., Pfüller, U., \& Scheffler, A. (2007). Solubility studies of oleanolic acid and betulinic acid in aqueous solutions and plant extracts of Viscum album L. Planta Medica, 73 , 157-162. http://dx.doi.org/10.1055/s-2007-967106 PMid:17415876

Kantamreddi, V. S. S., \& Wright, C. W. (2007). Investigation of Indian Diospyros species for antiplasmodial properties. eCAM, 1- 4.

Ko, H, Yu, S., Ko, F., Teng, C., \& Lin, C. (1997). Bioactive constituents of Morus australis and Broussonetia papyrifera. Journal of Natural Product, 60, 1008-1011. http://dx.doi.org/10.1021/np970186o PMid:9358644

Kokdil, G., Yalçin, S. M., \& Topçu, G. (1999). Nepetalactones and other constituents of Nepeta nuda ssp. Albiflora. Turkish Journal of Chemistry, 23, 99-104.

Krasutsky, P. A. (2006). Birch bark research and development. Natural Product Reports, 23(6), 919-942.

Lee, C. (1998). A new norlupene from the leaves of Melaleuca leucadendron. Journal of Natural Product, 61, 375-376. http://dx.doi.org/10.1021/np9606052 PMid:9548878

Lin, H., Ding, H., \& Wu, Y. (1998). Two novel compounds from Paeonia suffruticosa. Journal of Natural Product, 61(3), 343-346. PMid:9548873

Nattapong, S., \& Omboon, L. (2008). A new source of whitening agent from a Thai Mulberry plant and its betulinic acid quantitation. Natural Product Research, 22(9), 727-734. http://dx.doi.org/10.1080/14786410601130794 PMid:18569714

Nick, A., Wright, A. D., Rali, T., \& Sticher, O. (1995). Antibacterial triterpenoids from Dillenia papuana and their structure activity relationship. Phytochemistry, 40, 1691-1695. http://dx.doi.org/10.1016/0031-9422(95)00491-0

Pai, S. R. Nimbalkar, M. S., Pawar, N. V., \& Dixit, G. B. (2011). Optimization of extraction techniques and quantification of betulinic acid (BA) by RP-HPLC method from Ancistrocladus heyneanus Wall. Ex Grah. Industrial Crops and Products, 34, 1458-1464. http://dx.doi.org/10.1016/j.indcrop.2011.05.006

Pai, S. R., Upadhya, V., Hegde, H. V., Joshi, R. K., \& Kholkute, S. D. (2014). New report of triterpenoid betulinic acid (BA) along with oleanolic acid (OA) from Achyranthes aspera by RP-UFLC analysis and confirmation using HPTLC and FTIR techniques. Journal of Planar Chromatography - Modern TLC, 27(1), 38-41.

Pan, P., Jia, L. Y., \& Sun, Q. S. (2008). RP-HPLC determination of betulinic acid in Callicarpa macrophylla. Zhongguo Zhong Yao Za Zhi, 33(7), 753-755. PMid:18589770

Pereira, P. S., França, S. de C., de Oliveira, P. V. A., de Souza Breves, C. M., e Vaz Pereira, S. I., e Auro Nomizo, S. V. S., \& Dias, D. A.
(2008). Chemical constituents from Tabernaemontana catharinensis root bark: a brief NMR review of indole alkaloids and in vitro cytotoxicity. Quimica Nova, 31(1), 20-24. http://dx.doi.org/10.1590/S0100-40422008000100004

Pisha, E., Chai, H., Lee, I. S., Chagwedera, T. E., Farnsworth, N. R., Cordell, G. A., Beecher, C. W., Fong, H. H., Kinghorn, A. D., Brown, D. M., Wani, M. C., Wall, M. E., Hieken, T. J., Das Gupta, T. K., \& Pezzuto, J. M. (1995). Discovery of betulinic acid as a selective inhibitor of human melanoma that functions by induction of apoptosis. Nature Medicine, 1, 1046-1051. http://dx.doi.org/10.1038/nm1095-1046 PMid:7489361

Raskin, I., \& Ripoll, C. (2004). Can an apple a day keep the doctor away? Current Pharmaceutical Design, 10, 3419-3429. http://dx.doi.org/10.2174/1381612043383070 PMid:15544525

Razborek, M. I., Voncina, D. B., Dolecek, V., \& Voncina, E. (2007). Determination of major phenolic acids, phenolic diterpenes and triterpenes in Rosemary (Rosmarinus officinalis L.) by gas chromatography and mass spectrometry. Acta Chimica Slovenica, 54, 60-67.

Razborek, M. I., Voncina, D. B., Dolecek, V., \& Voncina, E. (2008). Determination of oleanolic, betulinic and ursolic acid in Lamiaceae and mass spectral fragmentation of their trimethylsilylated derivatives. Chromatographia, 67, 433-440. http://dx.doi.org/10.1365/s10337-008-0533-6

Recio, M. C., Giner, R. M., Manez, S., Gueho, J., Julien, H. R., Hostettmann, K., \& Rios, J. L. (1995). Investigations on the steroidal anti-inflammatory activity of triterpenoids from Diospyros leucomelas. Planta Medica, 61, 9-12. http://dx.doi.org/10.1055/s-2006-957988 PMid:7701004

Rosas, L. V., Cordeiro, M. S. C., Campos, F. R., Nascimento, S. K. R., Januário, A. H., França, S. C., Nomizo, A., Toldo, M. P. A., Albuquerque, S., \& Pereira, P. S. (2007). In vitro evaluation of the cytotoxic and trypanocidal activities of Ampelozizyphus amazonicus (Rhamnaceae). Brazilian Journal of Medical and Biological Research, 40, 663-670. http://dx.doi.org/10.1590/S0100-879X2007000500009 PMid:17464428

Saklani, A., \& Kutty, S. K. (2008). Plant derived compounds in clinical trials. Drug Discovery Today, 13(3-4), 161-171. http://dx.doi.org/10.1016/i.drudis.2007.10.010 PMid:18275914

Sousa, A. F., Pinto, P. C. R. O., Silvestre, A. J. D., \& Neto, C. P. (2006). Triterpenic and other lipophilic components from industrial cork byproducts. Journal of Agriculture and Food Chemistry, 54, 6888-6893. http://dx.doi.org/10.1021/jf060987 PMid:16939354

Takeoka, G., Dao, L., Teranishi, R., Wong, R., Flessa, S., Harden, L., \& Edwards, R. (2000). Identification of three triterpenoids in Almond Hulls. Journal of Agriculture and Food Chemistry, 48, 3437-3439. http://dx.doi.org/10.1021/if9908289 PMid:10956130

Taralkar, S. V, \& Chattopadhyay, S. (2012). A HPLC Method for determination of ursolic acid and betulinic acids from their methanolic extracts of Vitex Negundo Linn. Journal of Analytical and Bioanalytical Techniques, 3, 134. http://dx.doi.org/10.4172/2155-9872.1000134 
Woldemichael, G. M., Singh, M. P., Maiese, W. M., \& Timmermann, B. N. (2003). Constituents of antibacterial extract of Caesalpinia paraguariensis Burk. Zeitschrift fur Naturforschung, 58, 70-75. PMid:12622230

Yogeeswari, P. \& Sriram, D. (2005). Betulinic acid \& its derivatives: A review on their biological properties. Current Medicinal Chemistry, 12, 657-666. http://dx.doi.org/10.2174/0929867053202214 PMid:15790304

Zhang, Y., Yu, T., \& Wang, Y. (2003). Extraction of betulin from bark of Betula platyphylla by supercritical carbon dioxide extraction. Journal of Forestry Research, 14(3), 202-204. http://dx.doi.org/10.1007/BF02856830

Zhu, M., Phillipson, J. D., Greengrass, P. M., \& Bowery, N. G. (1996). Chemical and biological investigation of the root bark of Clerodendrum mandarinorum. Planta Medica, 62(5), 393-396. $\quad$ http://dx.doi.org/10.1055/s-2006-957923 PMid:9005449. 